\title{
TWO GENERATOR SUBALGEBRAS OF LIE ALGEBRAS
}

\author{
Kevin Bowman \\ Department of Physics, Astronomy and Mathematics \\ University of Central Lancashire \\ Preston PR1 2HE, England \\ David A. Towers \\ Department of Mathematics, Lancaster University \\ Lancaster LA1 4YF, England \\ and \\ Vicente R. Varea ${ }^{1}$ \\ Department of Mathematics, University of Zaragoza \\ Zaragoza, 50009 Spain
}

\begin{abstract}
In [14] Thompson showed that a finite group $G$ is solvable if and only if every twogenerated subgroup is solvable (Corollary 2, p. 388). Recently, Grunevald et al. [10] have shown that the analogue holds for finite-dimensional Lie algebras over infinite fields of characteristic greater than 5. It is a natural question to ask to what extent the two-generated subalgebras determine the structure of the algebra. It is to this question that this paper is addressed. Here, we consider the classes of strongly-solvable and of supersolvable Lie algebras, and the property of triangulability.
\end{abstract}

Keywords Lie algebra, two generator, solvable, supersolvable, triangulable.

AMS 2000 Mathematics subject classification 17B05, 17B30

\section{Introduction.}

Let $\mathcal{P}$ be a certain property that a subalgebra of a Lie algebra may possess. A task is to obtain information on the structure of a Lie algebra $L$ all of whose two-generated proper subalgebras possess the property $\mathcal{P}$. Given a subalgebra $S$ of $L$, we distinguish two types of properties that $S$ may possess: one type is that $S$ belong to a certain class $\mathcal{C}$ of Lie algebras, in this case the class $\mathcal{C}$ will be identified with the property; the other one is that $S$ be immersed in $L$ in a certain way. In this paper we will consider properties of these two types. Of the first type, we will consider the classes of strongly-solvable and supersolvable Lie algebras and of the other type the property that $S$ be triangulable on $L$.

In section 2, we give some preliminary results and we collect some known ones on important classes of Lie algebras. Most of them will be used in the sequel

In section 3, we consider the classes of strongly-solvable and of supersolvable Lie algebras. We prove that if $L$ is a solvable Lie algebra all of whose two-generated proper subalgebras are strongly

\footnotetext{
${ }^{1}$ Supported by DGI Grant BFM2000-1049-C02-01
} 
solvable (resp. supersolvable) then either $L$ is two-generated and every proper subalgebra of $L$ is strongly solvable (resp. supersolvable) or else $L$ is strongly solvable (resp. supersolvable). In the strongly-solvable case, we also prove that if $L$ is non-solvable, $F$ is infinite and $\operatorname{char}(\mathrm{F})>5$, then $L$ is two-generated, every proper subalgebra of $L$ is strongly solvable and $L / \phi(L)$ is simple (where $\phi(L)$ denotes the largest ideal of $L$ contained in every maximal subalgebra of $L$ ).

In section 4 , we prove that a solvable Lie algebra $L$ is triangulable whenever every two-generated proper subalgebra of $L$ is triangulable on $L$. Also, we prove that if every proper subalgebra of a Lie algebra $L$ is triangulable on $L$ but $L$ itself is not, then $L$ is two-generated and $L / \phi(L)$ is simple. Moreover, we give some information on the structure of a simple Lie algebra $L$ all of whose proper subalgebras are triangulable on $L$. In particular we obtain that such a Lie algebra is two-generated.

Throughout $L$ will denote a finite-dimensional Lie algebra over a field $F$. There will be no assumptions on $F$ other than those specified in individual results. We shall call $L$ supersolvable if there is a chain $0=L_{0} \subset L_{1} \subset \ldots \subset L_{n-1} \subset L_{n}=L$, where $L_{i}$ is an $i$-dimensional ideal of $L$. We shall call $L$ strongly solvable if its derived subalgebra $L^{2}$ is nilpotent. It is well known that

$$
\text { supersolvable } \Longrightarrow \text { strongly - solvable } \Longrightarrow \text { solvable }
$$

For algebraically closed fields of characteristic zero, these three classes of Lie algebras coincide (Lie's theorem). For fields of characteristic zero, every solvable Lie algebra is strongly solvable. There are well-known examples of solvable Lie algebras over algebraically closed fields of non-zero characteristic which are not supersolvable (see for instance [12, p.53] or [1]). Nevertheless, if the ground field $F$ is algebraically closed then every strongly-solvable Lie algebra is supersolvable (see [1, Lemma 2.4]).

A subalgebra $S$ of $L$ is said to be triangulable on $L$ if $\operatorname{ad}_{L} S=\left\{\operatorname{ad}_{L} x \mid x \in S\right\}$ is a Lie algebra of linear transformations of $L$ which is triangulable over the algebraic closure of $F$ (equivalently, if every element of $S^{2}$ acts nilpotently on $L$, see [23]). Some questions regarding triangulabilty of linear Lie algebras have been considered in [11].

The symbol $\dot{+}$ will denote a vector space direct sum. We say that $L$ is almost abelian if $L=$ $L^{2} \dot{+} F x$ with ad $x$ acting as the identity map on the abelian ideal $L^{2} ; L$ is quasi-abelian if it is abelian or almost abelian. Quasi-abelian Lie algebras are precisely those in which every subspace is a subalgebra.

\section{Preliminary Results}

The Frattini subalgebra of $L$, denoted by $F(L)$, is the intersection of all maximal subalgebras of $L$. It is known that $F(L)$ need not be an ideal of $L$, even for algebraically closed fields (see [18]); the Frattini ideal of $L$, denoted by $\phi(L)$, is the largest ideal of $L$ contained in $F(L)$. We say that $L$ is $\phi$ - free if $\phi(L)=0$. Clearly, $L / \phi(L)$ is $\phi$-free.

The following is straightforward. 
Lemma 2.1 A Lie algebra $L$ is two-generated if and only if $L / \phi(L)$ is two-generated.

A class $\mathcal{C}$ of Lie algebras is said to be saturated if $L \in \mathcal{C}$ whenever $L / \phi(L) \in \mathcal{C}$. It is wellknown that the classes of solvable, strongly-solvable, supersolvable and nilpotent Lie algebras are saturated (see [1] and [15])

For short, we will say that the property $\mathcal{P}$ satisfies condition $\left(^{*}\right)$ if for every Lie algebra $L$ all of whose two-generated proper subalgebras of $L$ possess the property $\mathcal{P}$, either $L$ itself possesses the property $\mathcal{P}$ or $L$ is two-generated. Next, we collect some known results on classes of Lie algebras which satisfy this condition.

\section{Theorem 0}

1. The classes of abelian, nilpotent and quasi-abelian Lie algebras satisfy condition $(*)$.

2. The class of simple (including the one-dimensional) Lie algebras satisfies condition $(*)$. If $\operatorname{char}(F) \neq 2,3$ and every two-generated proper subalgebra of $L$ is either simple or onedimensional, then every subalgebra of $L$ of dimension $>1$ is simple and $L$ is two-generated.

3. If $F$ is infinite and $\operatorname{char}(F)>5$, then the class of solvable Lie algebras satisfies condition $(*)$

Proof. (1): Clearly, the class of abelian Lie algebras satisfies condition $\left(^{*}\right)$. Now, suppose that every two-generated subalgebra of $L$ is nilpotent. Let $x \in L$ and choose any $y \in L$. Then $\langle x, y\rangle$ is nilpotent, so $y(\operatorname{ad} x)^{n}=0$ for some $n$. Hence $y(\operatorname{ad} x)^{d}=0$, where $d=\operatorname{dim} L$. This holds for all $y \in L$, so ad $x$ is nilpotent for all $x \in L$. It follows from Engel's Theorem that $L$ is nilpotent. To prove the last assertion in (1), suppose that every two-generated subalgebra of $L$ is quasi-abelian. Then every two-dimensional subspace of $L$ is a subalgebra of $L$, from which it follows that every subspace of $L$ is a subalgebra of $L$, and hence that $L$ is quasi-abelian.

(2): This is proved in [17, Proposition 3.2] and [20, Theorem 4].

(3): It is an immediate consequence of a result of Grunewald, Kunyavskii, Nikolova and Plotkin in [10].

The structure of solvable minimal non-abelian Lie algebras has been fully described by Stitzinger in [13, Theorem 1]. If $L$ is such a Lie algebra, then $L=A \dot{+} F x$, where $A$ is an abelian ideal of $L$ and ad $x$ acts irreducibly on $A$. Non-solvable minimal non-abelian Lie algebras have been studied by Farnsteiner in [5] and Gein in [6] (see also Elduque [3]).

We finish this section by collecting some known results on the structure of a minimal non-C Lie algebra for several classes $\mathcal{C}$.

Minimal non-quasi-abelian Lie algebras over a field of characteristic different from 2 and 3 have been studied by Gein in [7, Proposition 3] and Varea in [19, Theorem 2.2 and Corollary 2.4]. If 
$L$ is such a Lie algebra, then one of the following occurs: (a) $L$ is solvable minimal non-abelian; (b) $L \cong \operatorname{sl}(2)$; (c) $L$ is simple minimal non-abelian; or (d) $L$ has a basis $a_{1}, a_{2}, x$ with product given by one of the following rules:

1. $\left[a_{1}, a_{2}\right]=0,\left[a_{1}, x\right]=a_{1},\left[a_{2}, x\right]=\alpha a_{2}, 1 \neq \alpha \in F$

2. $\left[a_{1}, a_{2}\right]=0,\left[a_{1}, x\right]=a_{1},\left[a_{2}, x\right]=a_{1}+a_{2}$.

Minimal non-solvable Lie algebras over an algebraically closed field of prime characteristic have been studied by Varea in [21]

Minimal non-nilpotent Lie algebras have been studied by Stitzinger in [13], Gein and Kuznecov [9], Towers [16] and Farnsteiner [5]. In Gein [8], it is proved that if $L$ is simple and minimal non-nilpotent then the intersection of two distinct maximal subalgebras of $L$ is zero and $L$ has no non-zero ad-nilpotent elements. For the readers' convenience we include here a proof of Gein's result. (Our proof is different from the one given in [8].)

Theorem 2.2 (Gein [8]) Let $L$ be a simple Lie algebra over an arbitrary field F. Assume that every proper subalgebra is nilpotent. Then the following hold:

1. $M_{1} \cap M_{2}=0$ for every pair of different maximal subalgebras $M_{1}$ and $M_{2}$ of $L$;

2. L has no non-zero ad-nilpotent elements; and

3. $L$ is two-generated.

Proof. (1): Let $M$ be a maximal subalgebra of $L$. Assume that there exists a proper subalgebra $S$ of $L$ not contained in $M$ such that $M \cap S \neq 0$. Choose $S$ such that $\operatorname{dim} M \cap S$ is maximal. Nilpotency of $M$ implies that $N_{M}(M \cap S) \neq M \cap S$. Nilpotency of $S$ implies that $N_{S}(M \cap S) \neq$ $M \cap S$. Let $T$ be the subalgebra of $L$ generated by $N_{M}(M \cap S)$ and $N_{S}(M \cap S)$. Since $M \cap S$ is a non-zero ideal of $T$, it follows from the simplicity of $L$ that $T \neq L$. Moreover we have $S \cap M<T \cap M$, which contradicts our choice of $S$.

(2): Let $0 \neq x \in L$ be ad-nilpotent. Let $H$ be a maximal subalgebra of $L$ containing $x$. Let $L=L_{0} \dot{+} L_{1}$ be the Fitting decomposition of $L$ relative to $H$. Since $H$ is a maximal subalgebra of $L$, we have $H=L_{0}$. Since $x$ acts nilpotently on $L_{1}$, there exists $0 \neq y \in L_{1}$ such that $[x, y]=0$. Therefore $C_{L}(x)$ is not contained in $H$. Take a maximal subalgebra $M$ of $L$ containing $C_{L}(x)$. We find that $H \cap M \neq 0$ and $H \neq M$, which contradicts (1).

(3): Assume $L \neq<x, y>$ for every $x, y \in L$. Let $M$ be a maximal subalgebra of $L$. Take $0 \neq x \in M$ and $y \in L, y \notin M$. There exists a maximal subalgebra $S$ of $L$ containing $\langle x, y\rangle$. We have $S \cap M \neq 0$, which contradicts (1).

Conjecture: Every simple minimal non-solvable Lie algebra is two-generated. 
From [10] (see Theorem 0(3)) it follows that the conjecture is true for infinite fields of characteristic greater than 5 . In section 4 , we will prove that a Lie algebra $L$ all of whose proper subalgebras are triangulable on $L$ is two-generated.

\section{The classes of strongly-solvable and supersolvable Lie algebras}

In this section we consider the classes of strongly-solvable and supersolvable Lie algebras. We recall that the Lie algebra with basis $a, b, c$ and product given by $[a, b]=c,[a, c]=[b, c]=0$ is called the three-dimensional Heisenberg algebra.

The structure of solvable $\phi$-free minimal non-strongly-solvable Lie algebras was determined in $[2]$ and is given below.

Theorem 3.1 Let $L$ be a solvable $\phi$-free minimal non-strongly-solvable Lie algebra. Then $F$ has characteristic $p>0$ and $L=A \dot{+} B$ is a semidirect sum, where $A$ is the unique minimal ideal of $L, \operatorname{dim} A \geq 2, A^{2}=0$, and either $B=M \dot{+} F x$, where $M$ is an abelian minimal ideal of $B$ (type $I$ ), or $B$ is the three-dimensional Heisenberg algebra (type II).

By using Theorem 3.1 we prove the following.

Proposition 3.2 Let $L$ be solvable $\phi$-free minimal non-strongly-solvable Lie algebra. Then $L$ is two-generated.

Proof. The structure of $L$ is described in Theorem 3.1 above.

Suppose first that $L$ is of type I. Clearly, $[A, B]$ is an ideal of $L$. So that either $[A, B]=A$ or $[A, B]=0$. In the latter case, we have that $B$ is also an ideal of $L$, which is a contradiction. Therefore $[A, B]=A$. On the other hand, we have that $[x, M]$ is an ideal of $B$ contained in $M$. So, either $[x, M]=M$ or $[x, M]=0$. In the latter case, we have that $B$ is abelian. This yields that $L^{2} \leq A$ and hence $L^{2}$ is abelian, a contradiction. Therefore $[x, M]=M$. Then we have that $B^{2}=M$ and $L^{2}=A+B^{2}=A+M$. If $A(\operatorname{ad} m)^{2}=0$ for all $m \in M$ it is easy to see that $(\operatorname{ad} n)^{3}=0$ for all $n \in L^{2}$. But then $L^{2}$ is nil and hence nilpotent, by Engel's theorem, a contradiction. Choose $m \in M$ such that $A(\operatorname{ad} m)^{2} \neq 0$, and then $a \in A$ such that $[[a, m], m] \neq 0$. Now $B=<m, x>$. Put $D=<a+x, m>$. Then $[[a+x, m], m]=[[a, m], m] \in D$, whence $D \cap A \neq 0$. Clearly $L=A+D$ so $D \cap A$ is an ideal of $L$. It follows that $A \subseteq D$, giving $D=A+<x, m\rangle=L$ and $L$ is two-generated.

So suppose that $L$ is of type II. Then $B$ has a basis $c, s, x$ with product given by $[s, x]=c$, $[c, x]=[c, s]=0$. Put $C=C_{A}(c)$. Then $C$ is an ideal of $L$ and so $C=A$ or $C=0$. The former implies that $L^{2}=A+F c$ is abelian, a contradiction, so $C=0$. Let $D=\langle a+s, x\rangle$. Then $[a, x]+c \in D$. If $[a, x]=0$, then $c \in D$. But this implies that $0 \neq[a, c] \in D \cap A$, whence $L=D$ 
as above. So suppose that $[a, x] \neq 0$. This yields that $[[a, x], x] \in D$, from which $[[a, x], x] \neq 0$ would give $L=D$ again. So we have that $[a, x] \neq 0$ but $[[a, x], x]=0$. Put $E=<[a, x]+s, x>$. Then $c=[[a, x]+s, x] \in E$, whence $[[a, x], c] \in E$. But $[[a, x], c] \neq 0$ since $[a, x] \neq 0$, giving $E \cap A \neq 0$ from which $L=E$. The proof is complete.

Now we are able to prove the following result.

Theorem 3.3 Let L be a Lie algebra such that every two-generated proper subalgebra is strongly solvable.

1. Assume that $L$ is solvable but not strongly solvable. Then every proper subalgebra of $L$ is strongly solvable and $L$ is two-generated.

2. Assume that $L$ is non-solvable, $F$ is infinite and $\operatorname{char}(F)>5$. Then $L$ is two-generated, every proper subalgebra of $L$ is strongly solvable and $L / \phi(L)$ is simple.

Proof. (1): Let $S$ be a non-strongly-solvable subalgebra of $L$ of minimal dimension. Clearly, $S$ is minimal non-strongly-solvable. We have that $S / \phi(S)$ is $\phi$-free, solvable and minimal nonstrongly-solvable. By Proposition 3.2 it follows that $S / \phi(S)$ is two-generated, whence $S$ is also two-generated, by Lemma 2.1. By our hypothesis it follows that $S=L$. This yields that $L$ is minimal non-strongly-solvable and two-generated. The proof of (1) is complete.

(2): By using Theorem 0(3) we obtain that every non-solvable subalgebra of $L$ (including $L$ itself) is two-generated. From this and our hypothesis it follows that every proper subalgebra of $L$ is solvable. Now let $S$ be a proper subalgebra of $L$. Assume that $S$ is not strongly solvable. Then, by (1) it follows that $S$ is two-generated. But then, by our hypothesis $S$ is strongly solvable, a contradiction. Put $\bar{L}=L / \phi(L)$. We have that $\bar{L}$ is non-solvable but every proper subalgebra of $\bar{L}$ is solvable. On the other hand, since $\bar{L}$ is $\phi$-free we have that $\bar{L}=N+S$, $N \cap S=0$, where $N$ is the largest nilpotent ideal of $\bar{L}$ and $S$ is a non-solvable subalgebra of $\bar{L}$. This yields that $S=\bar{L}$ and hence $N=0$. Let $A$ be a minimal ideal of $\bar{L}$. We have that either $A^{2}=A$ or $A^{2}=0$. In the latter case, we have $A \leq N=0$, a contradiction. This yields that $A=A^{2}$ and hence $A=\bar{L}$. Therefore $\bar{L}$ is simple. This completes the proof.

Next we consider the class of supersolvable Lie algebras

The structure of strongly-solvable minimal non-supersolvable Lie algebras as well as that of $\phi$ free, non-strongly-solvable and minimal non-supersolvable Lie algebras were determined in [4]. For the readers' convenience, we give them below

Theorem 3.4 [4, Theorems 1.1 and 1.2] Let L be minimal non-supersolvable.

1. If $L$ is strongly solvable, then $L=\phi(L) \dot{+} A \dot{+} F x$, where $A$ is subspace of $L, A^{2} \leq \phi(L)$, with $\operatorname{ad} x$ acting irreducibly on $A$ and $\left.\operatorname{ad} x\right|_{\phi(L)}$ is split. 
2. If $L$ is $\phi$-free and non-strongly-solvable, then $\operatorname{char}(F)=p>0$ and one of the following hold:

(a) $L=\left(\left(x, y, e_{0}, e_{1}, \ldots, e_{p-1}\right)\right)$ with $\left[e_{i}, y\right]=(\alpha+i) e_{i}$ where $\alpha$ is a fixed scalar in $F$, $\left[e_{i}, x\right]=e_{i+1}$ (indices mod.p), $[x, y]=x,\left[e_{i}, e_{j}\right]=0$ and $F=\left\{t^{p}-t \mid t \in F\right\}$.

(b) $L=\left(\left(x, y, z, e_{0}, e_{1}, \ldots, e_{p-1}\right)\right)$ with $\left[e_{i}, z\right]=e_{i}$ for every $i$ and $\left[e_{i}, x\right]=e_{i+1}(i=$ $0, \ldots, p-2)$ (non-specified products are zero) and $F$ is perfect whenever $p=2$.

Now we can prove the following

Theorem 3.5 Let L be a solvable Lie algebra.

1. If $L$ minimal non-supersolvable, then $L$ is two-generated.

2. If every two-generated proper subalgebra of $L$ is supersolvable but $L$ is not supersolvable, then every proper subalgebra of $L$ is supersolvable. So, either $L$ has the structure given in (1) of Theorem 3.4 or $L / \phi(L)$ is isomorphic to one of the Lie algebras described in (2) of Theorem 3.4.

Proof. (1): If $L^{2}$ is not nilpotent, then $L$ is minimal non-strongly-solvable. So, by Theorem 3.3 it follows that $L$ is two-generated. Now assume that $L^{2}$ is nilpotent. Put $\bar{L}=L / \phi(L)$. We have that $\bar{L}$ is $\phi$-free, strongly solvable and minimal non-supersolvable. By Theorem 3.4, we have that $\bar{L}=A \dot{+} F x$ where $A$ is a nonzero abelian ideal of $\bar{L}$ and ad $x$ acts irreducibly on $A$. Pick $0 \neq a \in A$. We have that $\bar{L}$ is generated by $a$ and $x$. From Lemma 2.1 it follows that $L$ is two-generated. The proof of (1) is complete.

(2): Clearly, $L$ has a subalgebra $S$ which is minimal non-supersolvable. From (1) it follows that $S$ is two-generated. Then, by our hypothesis, we have that $S=L$.

\section{The property of triangulability}

A subalgebra $S$ of $L$ is said to be triangulable on $L$ if $\operatorname{ad}_{L} S=\left\{\operatorname{ad}_{L} x \mid x \in S\right\}$ is a Lie algebra of linear transformations of $L$ which is triangulable over the algebraic closure of $F$. A subalgebra $S$ of $L$ is said to be nil on $L$ if $\operatorname{ad} x$ is nilpotent for every $x \in S$. It is well-known that for every subalgebra $S$ of $L$, there is a unique maximal ideal $\operatorname{nil}(S)$ of $S$ consisting of ad-nilpotent elements of $L$ (see [23, Proposition 2.1]). Also, it is known that $S$ is triangulable on $L$ if and only if $S / \operatorname{nil}(S)$ is abelian (see [23, Theorem 2.2]). Note that every subalgebra of $L$ which is triangulable on $L$ is strongly solvable.

First, we give the following easy lemma which will be used in the sequel. 
Lemma 4.1 Let $L$ be a Lie algebra and $S$ and $T$ subalgebras of $L$ which are nil on $L$. Assume that $[S, T] \subset T$. Then $S+T$ is nil on $L$.

Proof. We see that the set ad $S \cup \operatorname{ad} T$ is weakly closed in the sense of Jacobson [12]. Therefore, by [12, Theorem 1,p.33], every element of $S+T$ acts nilpotently on $L$.

Proposition 4.2 Let $S$ be a triangulable subalgebra of $L$. Then $\operatorname{nil}(S)$ is precisely the set of all elements of $S$ which act nilpotently on $L$.

Proof. Let $\Omega$ be an algebraic closure of $F$ and let $L_{\Omega}=L \otimes_{F} \Omega$. Put $A=\operatorname{ad}_{L} S$. We have that $A \leq g l(L)$ and that $A_{\Omega}$ is a subalgebra of $g l\left(L_{\Omega}\right)$ and a set of simultaneously triangulable linear maps. Then it is obvious that the set $N\left(A_{\Omega}\right)$ of nilpotent elements of $A_{\Omega}$ is closed under linear combinations and that $\left(A_{\Omega}\right)^{2}<N\left(A_{\Omega}\right)$. Since $\left(A^{2}\right)_{\Omega}=\left(A_{\Omega}\right)^{2}$, it follows that $A^{2}$ is contained in the set $N$ of nilpotent elements in $A$. Also, we have that $N$ is closed under linear combinations. So $N$ is actually an ideal of $A$. Then $\operatorname{nil}(S)$ is contained in the inverse image $N^{\prime}$ of $N$ under the adjoint representation $\operatorname{ad}_{L}$. This is a Lie-homomorphism, whence $N^{\prime}$ is an ideal of $S$. It follows that $N^{\prime}=\operatorname{nil}(S)$. This completes the proof.

Lemma 4.3 Let $L$ be a Lie algebra which is not two-generated. Then for each maximal subalgebra $M$ of $L$ and each non-zero element $x$ in $M$ there exists a maximal subalgebra $S$ of $L$ different from $M$ containing $x$. If the ground field $F$ is infinite, then each element of $L$ lies in infinitely many maximal subalgebras of $L$.

Proof. Let $M$ be a maximal subalgebra of $L$ and $0 \neq x \in M$. Pick $y \in L \backslash M$. By hypothesis, $\langle x, y\rangle \neq L$. So, there exists a maximal subalgebra $S$ of $L$ containing $\langle x, y\rangle$. We see that $S \neq M$ since $y \in S$ and $y \notin M$. Now suppose that $F$ is infinite. Let $x \in L$ and assume that $M_{1}, \cdots, M_{r}$ are the only maximal subalgebras of $L$ which contain $x$. Since $F$ is infinite, $M_{1} \cup \cdots \cup M_{r} \neq L$. Pick $y \in L, y \notin M_{1} \cup \cdots \cup M_{r}$. Then, by hypothesis $\left.L \neq<x, y\right\rangle$. Take a maximal subalgebra $S$ of $L$ containing $\langle x, y\rangle$. We see that $x \in S$ and $S \neq M_{i}$ for every $i$, which is a contradiction.

Now we give some information on the structure of a simple Lie algebra all of whose proper subalgebras are triangulable. Clearly, a simple minimal non-abelian Lie algebra satisfies this condition. However, we do not know any other example.

Theorem 4.4 Let $L$ be a simple Lie algebra such that every proper subalgebra of $L$ is triangulable on L. Then the following hold:

1. if $M$ is a maximal subalgebra of $L$, then either $M$ is abelian and none of the non-zero elements of $M$ acts nilpotently on $L$ or $\operatorname{nil}(M)$ is a non-zero maximal nil subalgebra of $L$; 
2. if $K$ is a non-zero maximal nil subagebra of $L$, then the normalizer of $K$ in $L$ is a maximal subalgebra of $L$;

3. for each two different maximal subalgebras $M_{1}$ and $M_{2}$ of $L$, none of the non-zero elements of $M_{1} \cap M_{2}$ acts nilpotently on $L$ (in particular, $M_{1} \cap M_{2}$ is abelian); and

4. $L$ is two-generated.

Proof. (1): Let $M$ be any maximal subalgebra of $L$. First assume that $\operatorname{nil}(M)=0$. Then we have $M^{2} \leq \operatorname{nil}(M)=0$. So, $M$ is abelian. By Proposition 4.2 it follows that $M$ does not contain any non-zero ad-nilpotent element of $L$. Now assume $\operatorname{nil}(M) \neq 0$. Let $K$ be a maximal nil subalgebra of $L$ containing $\operatorname{nil}(M)$. Assume $K \neq \operatorname{nil}(M)$. By Proposition 4.2 again, we have $M \cap K=\operatorname{nil}(M)$. Nilpotency of $K$ implies that nil $(M)$ is properly contained in the normalizer $N_{K}(\operatorname{nil}(M))$ of $\operatorname{nil}(M)$ in $K$. This yields that nil $(M)$ is an ideal of $L$, since $L=M+N_{K}(\operatorname{nil}(M))$ by maximality of $M$, a contradiction. Therefore $K=\operatorname{nil}(M)$ and so $\operatorname{nil}(M)$ is maximal nil on $L$. The proof of (1) is complete.

(2): Let $0 \neq K$ be a maximal nil subalgebra of $L$. Let $M$ be a maximal subalgebra of $L$ containing $K$. Then, by Proposition 4.2 it follows that $K=\operatorname{nil}(M)$. This yields that $M=N_{L}(K)$ and hence $N_{L}(K)$ is a maximal subalgebra.

(3): Let $M_{1}, M_{2}$ be distinct maximal subalgebras of $L$. By Proposition 4.2 we have that

$$
\operatorname{nil}\left(M_{1} \cap M_{2}\right)=\operatorname{nil}\left(M_{1}\right) \cap \operatorname{nil}\left(M_{2}\right)
$$

We need to prove that $\operatorname{nil}\left(M_{1} \cap M_{2}\right)=0$. Assume that $\operatorname{nil}\left(M_{1} \cap M_{2}\right) \neq 0$. Choose $M_{1}$ and $M_{2}$ such that the dimension of $\operatorname{nil}\left(M_{1} \cap M_{2}\right)$ is maximal. Suppose that $\operatorname{nil}\left(M_{1} \cap M_{2}\right)=\operatorname{nil}\left(M_{2}\right)$. Then we have $0 \neq \operatorname{nil}\left(M_{2}\right) \leq \operatorname{nil}\left(M_{1}\right)$. By (1) it follows that $\operatorname{nil}\left(M_{2}\right)=\operatorname{nil}\left(M_{1}\right)$. This yields that nil $\left(M_{2}\right)$ is an ideal of $M_{1}$ and $M_{2}$ and therefore it is an ideal of $L$. Simplicity of $L$ implies that $\operatorname{nil}\left(M_{2}\right)=0$, a contradiction. Hence $\operatorname{nil}\left(M_{1} \cap M_{2}\right) \neq \operatorname{nil}\left(M_{2}\right)$. Analogously, we have $\operatorname{nil}\left(M_{1} \cap M_{2}\right) \neq \operatorname{nil}\left(M_{1}\right)$. Now, by using Engel's Theorem we obtain subalgebras $S_{1}$ and $S_{2}$ of $L$ strictly containing $\operatorname{nil}\left(M_{1} \cap M_{2}\right)$ and such that

$$
\operatorname{nil}\left(M_{1} \cap M_{2}\right) \triangleleft S_{i} \leq \operatorname{nil}\left(M_{i}\right) \text { for } i=1,2
$$

Let $S$ be the subalgebra of $L$ generated by $S_{1}$ and $S_{2}$. We find that $\operatorname{nil}\left(M_{1} \cap M_{2}\right)$ is an ideal of $S$. Simplicity of $L$ implies that $S \neq L$. Take a maximal subalgebra $M_{3}$ of $L$ containing $S$. We have

$$
\operatorname{nil}\left(M_{1} \cap M_{2}\right) \subset S_{1} \subseteq M_{3} \cap \operatorname{nil}\left(M_{1}\right)=\operatorname{nil}\left(M_{1} \cap M_{3}\right)
$$

which contradicts the maximality of $\operatorname{nil}\left(M_{1} \cap M_{2}\right)$. This completes the proof of (3).

(4): Assume that $L \neq<a, b>$ for every $a, b \in L$. Let $K$ be a nil subalgebra of $L$ of maximal dimension. If $K=0$, then by (1) we have that every maximal subalgebra of $L$ is abelian. Let $M_{1}, M_{2}$ be distinct maximal subalgebras of $L$. We have that $M_{1} \cap M_{2}=0$; since, otherwise, we would have that $M_{1} \cap M_{2}$ is a nonzero ideal of $L$, which is a contradiction. Pick $0 \neq a_{1} \in M_{1}$ and $0 \neq a_{2} \in M_{2}$. Since $<a_{1}, a_{2}>\neq L$, we can take a maximal subalgebra $M_{3}$ of $L$ containing 
$a_{1}$ and $a_{2}$. But then we have $0 \neq a_{1} \in M_{1} \cap M_{3}$ and $M_{1} \neq M_{3}$, a contradiction. Therefore $K \neq 0$. Pick $x \in K, x \neq 0$. By (3), there exists only one maximal subalgebra of $L$ containing $x$, which contradicts Lemma 4.3. Therefore $L$ can be generated by two elements. The proof of the theorem is complete.

Now we can prove the following

Lemma 4.5 1. If $\phi(L) \leq S \leq L$, then $\operatorname{nil}(S / \phi(L))=\operatorname{nil}(S) / \phi(L)$.

2. If every two-generated proper subalgebra of $L$ is triangulable on $L$, then the same holds in $L / \phi(L)$.

Proof. (1): Assume $\phi(L) \leq S \leq L$. Let $\operatorname{nil}(S / \phi(L))=K / \phi(L)$. Since $\phi(L)$ is a nilpotent ideal of $L$ (see [15]), it is nil on $L$. So, $\phi(L) \leq \operatorname{nil}(S)$. Clearly, $\operatorname{nil}(S) \leq K$. Now let $x \in K$. We have that $x$ acts nilpotently on $L / \phi(L)$. This yields that $L=\phi(L)+L_{0}(x)$, where $L_{0}(x)$ is the Fitting null-component of $L$ relative to ad $x$. As $L_{0}(x)$ is a subalgebra of $L$, it follows that $L=L_{0}(x)$. So that $x$ acts nilpotently on $L$. This yields that $K \leq \operatorname{nil}(S)$.

(2): Assume that every two-generated proper subalgebra of $L$ is triangulable on $L$. Let $S / \phi(L)$ be a two-generated proper subalgebra of $L / \phi(L)$. Take elements $a, b \in L$ such that $S / \phi(L)=<$ $a+\phi(L), b+\phi(L)\rangle$. We have that $S=<a, b\rangle+\phi(L)$ and that $\langle a, b\rangle$ is triangulable on $L$. On the other hand, from Lemma 4.1 it follows that $\phi(L)+\operatorname{nil}(<a, b\rangle)$ is nil on $L$. Since $\phi(L)+\operatorname{nil}(<a, b\rangle)$ is an ideal of $S$, we have that $\phi(L)+\operatorname{nil}(<a, b\rangle) \leq \operatorname{nil}(S)$. Then,

$$
S^{2} \leq \phi(L)+<a, b>^{2} \leq \phi(L)+\operatorname{nil}(<a, b>) \leq \operatorname{nil}(S)
$$

So, we have that

$$
(S / \phi(L))^{2}=S^{2}+\phi(L) / \phi(L) \leq \operatorname{nil}(S) / \phi(L)=\operatorname{nil}(S / \phi(L))
$$

by (1). Therefore $S / \phi(L)$ is triangulable on $L / \phi(L)$. The proof is complete.

Theorem 4.6 1. If $L$ is solvable and every two-generated proper subalgebra of $L$ is triangulable on $L$, then $L$ is triangulable.

2. If every proper subalgebra of $L$ is triangulable on $L$ but $L$ itself is not, then $L$ is twogenerated and $L / \phi(L)$ is simple (so, $L / \phi(L)$ satisfies (1)-(4) in Theorem 4.4).

Proof. (1): Let $L$ be solvable and every two-generated proper subalgebra of $L$ be triangulable on $L$. In particular, we have that every two-generated proper subalgebra of $L$ is strongly solvable. Put $\bar{L}=L / \phi(L)$. Then, by Theorem 3.3(1) it follows that every proper subalgebra of $\bar{L}$ is strongly solvable. Moreover, by Lemma 4.5 we have that every two-generated proper subalgebra of $\bar{L}$ is triangulable on $\bar{L}$. Now suppose that $L$ is not triangulable. So that $L$ is not strongly solvable. Thus, $\bar{L}$ is not strongly solvable either. By using Theorem 3.1 we obtain that $\bar{L}=A+B$, 
where $A$ is the unique minimal ideal of $\bar{L}, A^{2}=0, B<\bar{L}, A \cap B=0$, and either $B=M \dot{+} F x$, where $M$ is the unique minimal ideal of $B$ or $B$ is the three-dimensional Heisenberg algebra. We have that $\bar{L}^{2}=A+B^{2}$. On the other hand, we see that $B$ is two-generated. So, $B$ is triangulable on $\bar{L}$. This yields that $B^{2}$ acts nilpotently on $A$ and therefore $\bar{L}^{2}$ is nilpotent. So that $\bar{L}$ is strongly solvable. This contradiction completes the proof of (1).

(2): Assume that every proper subalgebra of $L$ is triangulable on $L$ but $L$ itself is not. By (1) we have that $L$ is not solvable. By Lemmas 4.5(1), 2.1 and since the class of solvable Lie algebras is saturated, we may suppose without loss of generality that $L$ is $\phi$-free. By Towers [15] we have that $L=N+S$ and $N \cap S=0$, where $N$ is the largest nilpotent ideal of $L$ and $S$ is a subalgebra of $L$. If $S \neq L$, then we have that $S$ is solvable. So, $L$ is solvable, a contradiction. It follows that $N=0$ and so $L$ is simple. Hence, $L$ satisfies (1)-(4) in Theorem 4.4. The proof is complete.

\section{ACKNOWLEDGMENT}

The authors are grateful to the referee for his/her useful comments; in particular, for providing a shorter and clearer proof of Proposition 4.2.

\section{References}

[1] D.W. Barnes And M.L. Newell, Some theorems on saturated homomorphs of soluble Lie algebras. Math. Zeit. 115 (1970) 179-187.

[2] K. Bowman and D.A. Towers, On almost nilpotent-by-abelian Lie Algebras, Lin. Algebra Appl. 247 (1996) 159-167.

[3] A. Elduque, A note on noncentral simple minimal nonabelian Lie algebras, Comm. in Algebra, 15(7) (1987) 1313-1318.

[4] A. Elduque and V.R. Varea, Lie algebras all of whose subalgebras are supersolvable, Can. Math. Soc. Conf. Proc. 5 (1986) 209-218.

[5] R. Farnsteiner, On ad-semisimple Lie algebras, J. Algebra, 83 (1983) 510-519.

[6] A.G. GeIn, Minimal noncommutative and minimal nonabelian algebras, Comm. in Algebra, 13(2) (1985) 305-328.

[7] A.G. Gein, Modular rule and relative complements in the lattice of subalgebras of a Lie algebra, Sov. Math., 31 (3) (1987) 22-32; Translation from Izv. Vyssh. Uchebn. Zaved. Mat., 3 (298) (1987) 18-25

[8] A.G. Gein, Lie algebras with constraints on subalgebras (Russian). Ural U. Sverdlovsk 1989.

[9] A.G. Gein and S.V. Kuznecov, Minimal non-nilpotent Lie algebras, Ural. Gos. Univ. Mat. Zap., 8(tetrad 3) (1972) 18-27. 
[10] F. Grunewald, B. Kunyavskit, D. Nikolova, and E. Plotkin, Two-variable identities in groups and Lie algebras, Zap. Nauch. Semin. POMI, 272 (2000) 161-176; J. Math. Sci. (New York), 116 (2003) 2972-2981.

[11] R. Guralnick, Triangularization of sets of matrices, Linear Multilinear Algebra, 9 (1980), $133-140$.

[12] N. Jacobson, Lie algebras. New York: Dover Publ. 1979

[13] E.L. Stitzinger, Minimal non-nilpotent solvable Lie algebras, Proc. Amer. Math. Soc., 28 (1971) 47-49.

[14] J. Thompson, Non-solvable finite groups all of whose local subgroups are solvable, Bull. Amer. Math. Soc., 74 (1968) 383-437.

[15] D.A. Towers, A Frattini theory for algebras, Proc. London Math. Soc. (3), 27 (1973), 440-462.

[16] D.A. Towers, Lie algebras all of whose proper subalgebras are nilpotent, Lin. Algebra Appl., 32 (1980) 61-73.

[17] V.R. VAREA, Lie algebras none of whose Engel subalgebras are in intermediate position, Comm. in Algebra, 15(12) (1987), 2529-2543.

[18] V.R. VAREA, Modular subalgebras, Quasi-ideals and inner ideals in Lie algebras of prime characteristic, Comm. in Algebra, 21(11), (1993) 4195-4218.

[19] V.R. VAREA, Lie algebras whose proper subalgebras are either semisimple, abelian or almost-abelian, Hiroshima Math. J., 24 (1994), 221-241.

[20] V.R. VAREa, Supersimple and upper semimodular Lie algebras, Comm. in Algebra, 23(6) (1995), 2323-2330.

[21] V.R. VAREA, Lie algebras all of whose proper subalgebras are solvable, Comm. in Algebra, 23(9) (1995), 3245-3267.

[22] D.J. Winter, Abstract Lie Algebras, MIT Press: Cambridge, Mass., 1972.

[23] D.J. Winter, Cartan Decompositions and Engel subalgebra Triangulability, J. Algebra, 62 (1980), 400-417. 